Jones, S.E. and J. Ellsworth. Geo-fusion and Cold Nucleosynthesis. in Tenth International Conference on Cold Fusion. 2003. Cambridge, MA: LENR-CANR.org. This paper was presented at the 10th International Conference on Cold Fusion. It may be different from the version published by World Scientific, Inc (2003) in the official Proceedings of the conference.

\title{
Geo-fusion and Cold Nucleosynthesis
}

\author{
S. E. Jones with J. E. Ellsworth \\ Department of Physics and Astronomy, Brigham Young University, Provo, UT 84604
}

\begin{abstract}
In our 1986 and1989 papers, we discussed the hypothesis of cold nuclear fusion in condensed matter and particularly in the planets. ${ }^{1,2}$ The purpose of this paper is to provide an update on geo-fusion research, then to consider an important extension of the cold-fusion idea: "cold nucleosynthesis" in condensed matter. Cold nucleosynthesis experiments are underway at Brigham Young University.
\end{abstract}

\section{$\underline{\text { Introduction }}$}

The hypothesis of natural fusion occurring within the earth (geo-fusion) goes back to 198586. In our original 1985 submission on fusion in condensed matter, Clinton Van Siclen and I discussed "whether piezonuclear fusion within the liquid metallic hydrogen core of Jupiter can account for the excess heat radiated from the planet." The paper was published in March 1986, and that same month I gave an invited colloquium at Brigham Young University on this subject of piezonuclear fusion. BYU Professor Paul Palmer made the extension of our ideas to the earth, that is, he suggested that "cold fusion" might occur deep within the earth. We immediately began a collaboration including Bart Czirr at BYU and others, to test the idea that nuclear reactions might somehow be enhanced in metal deuterides. We initiated original experiments in the BYU underground laboratory in April 1986. (It was not until years later that we learned of somewhat parallel work of Stanley Pons and Martin Fleischmann, and also of Andrei Lipson and his team in Russia.) In our publication in Nature in 1989, we emphasized "Observations of naturally occurring ${ }^{3} \mathrm{He}$ in the earth suggested to us new directions for laboratory investigations of nuclear fusion in condensed matter."2 We also noted that tritium production from d-d fusion was a possible product of geo-fusion.

Here we provide an update on geo-fusion research, then discuss an extension of the coldfusion idea -- "cold nucleosynthesis" in condensed matter. 
$\underline{\text { Volcanic Tritium Measurements as a Test for Natural Fusion in the Earth }}$

Our experimental evidence shows fusion products from deuterium-bearing metals under non-equilibrium conditions. ${ }^{2,3}$ We believe that similar conditions found deep inside the earth may result in natural $\mathrm{d}-\mathrm{d}$ and $\mathrm{p}-\mathrm{d}$ fusion reactions, generating ${ }^{3} \mathrm{He}$ and ${ }^{3} \mathrm{H}$ (tritium):

$$
\begin{aligned}
& d+d \rightarrow p(3.02 \mathrm{MeV})+\mathrm{t}(1.01 \mathrm{MeV}) \\
& \mathrm{d}+\mathrm{d} \rightarrow \mathrm{n}(2.45 \mathrm{MeV})+{ }^{3} \mathrm{He}(0.82 \mathrm{MeV}) .
\end{aligned}
$$

${ }^{3} \mathrm{He}$ is already known to be released by many volcanoes, but this could be due to ${ }^{3} \mathrm{He}$ stored during the earth's formation. ${ }^{4}$ Since tritium decays with a half-life of 12.4 years, a good test of our hypothesis would be the detection of tritium emanating from deep within the earth and found in magmatic gases and fluids.

Fortunately, extensive tritium measurements of volcanic products were taken a dozen years ago, following on predictions of our 1989 paper. $^{5}$ Goff and McMurtry noted that "prevailing wisdom assumes that ${ }^{3} \mathrm{H}$ (magma) is essentially 0. " Furthermore, ${ }^{3} \mathrm{H}$ production in magmas due to fission and radioactive decay was calculated to be very small, $<0.001$ Tritium Units (T.U.) for nearly all volcanoes. It was further established that "Pre-bomb levels of ${ }^{3} \mathrm{H}$ in precipitation ... are about 1 T.U. for Hawaii." Unfortunately, anthropogenic ${ }^{3} \mathrm{H}$ from hydrogenbomb testing four decades ago caused meteoric (from precipitation) ${ }^{3} \mathrm{H}$ to rise, making the determination of magmatic ${ }^{3} \mathrm{H}$ much more difficult. The authors carefully disentangled data to assess magmatic tritium from various volcanoes and found that magmatic tritium values were generally small/consistent with zero, as summarized in Figure $1 .{ }^{5}$

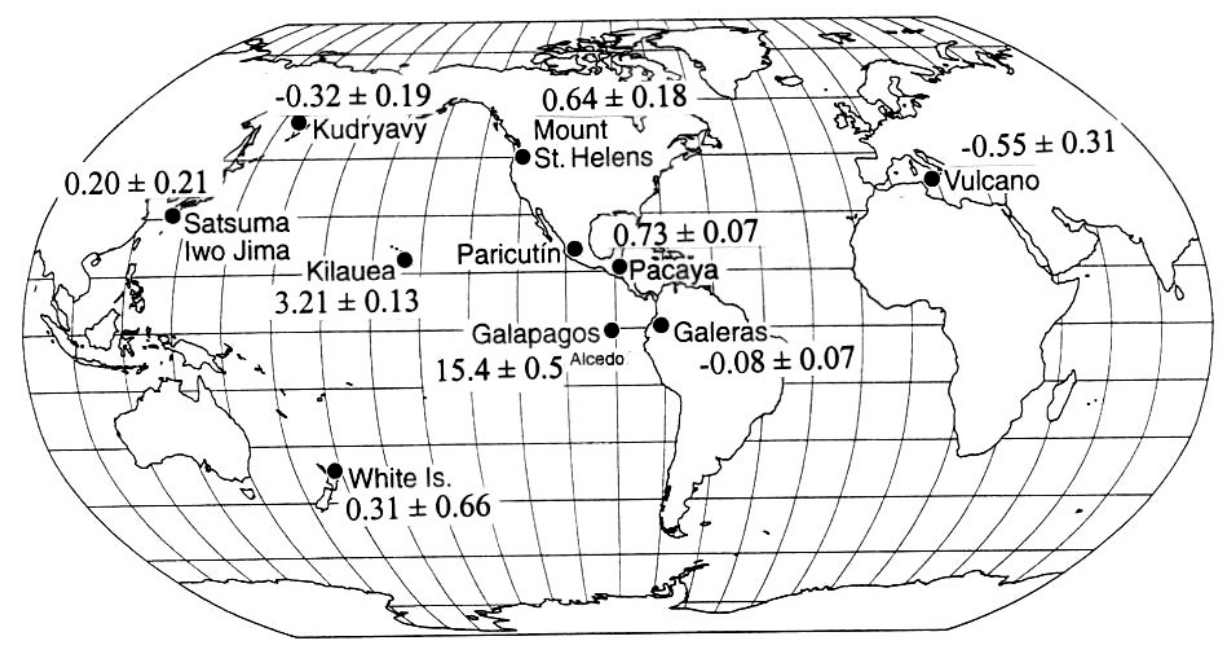

Fig. 1 World map showing volcanoes studied, modified from Ref. 5. Tritium activity (in Tritium Units, 1 T.U. $=3.2 \mathrm{pCi} / \mathrm{kg} \mathrm{H}_{2} \mathrm{O}$ ) evaluated for end-member magmatic water is also displayed. ${ }^{5}$

However, there were notable "positive ${ }^{3} \mathrm{H}$ anomalies" among the ten volcano samples: Kilauea in Hawaii and Alcedo geyser in the Galapogos Archipelago. ${ }^{5}$ The magmatic-tritium result for Kilauea is clearly not zero: the tritium content of end-member magmatic fluid was found to be $(3.21 \pm 0.13)$ tritium units (T.U.) "Kilauea fumarole condensates have high... ${ }^{3} \mathrm{H}$ 
contents (as much or more than 1990-1991 rain)". 5 In addition, a striking (15.4 \pm 0.5$)$ T.U. was discovered in the Alcedo "geyser". Although it was not possible for the authors to determine the end-member magmatic content in this case, this value appears far above that expected from rain (meteoric) water.

We would like to call attention to the fact that these two volcanoes are hot spot volcanoes. That is, these volcanoes are produced by magma from plumes which rise hundreds of miles from the core-mantle boundary. The other volcanoes shown on the data summary map (Fig. 1) were not hot-spot volcanoes, tapping instead much shallower magmas. Hot spot volcanoes always show high ${ }^{3} \mathrm{He} /{ }^{4} \mathrm{He}$ ratios, typically tens to hundreds of times higher than in volcanoes found at subduction zones. Thus, both tritium and ${ }^{3} \mathrm{He} /{ }^{4} \mathrm{He}$ ratios are low for subducting zone volcanoes, yet anomalously high for the hot spot volcano Kilauea and, evidently, Alcedo "geyser". We suggest the following hypothesis, building on our earlier geofusion hypothesis: ${ }^{2}$

Natural geo-fusion in the earth occurs in or near the core of the earth, in the hot, hydrogen-bearing metals and minerals which are subjected to extreme off-equilibrium conditions deep in the earth. This hypothesis can be tested by measuring tritium and helium-3 in magmatic fluids from hot-spot volcanoes which tap magmas from plumes arising from the core-mantle boundary. In particular, magmatic waters of Kilauea, Loihi, and Icelandic volcanoes are predicted to contain significant tritium. We predict that tritium is also present in Jupiter, originating from "cold" fusion in or near its metallic hydrogen core.

It appears to us that important questions related to fusion-generated tritium in the planets remain open. About a dozen years have now elapsed since the baseline data ${ }^{5}$ were taken at Kilauea, approximately a full tritium half-life. So we strongly urge a follow-up study there to determine whether tritium levels have dropped dramatically - as must be the case if the "anomalous" ${ }^{3} \mathrm{H}$ measured in the excellent previous survey were simply anthropogenic. Detection of significant tritium in Loihi and Icelandic volcanoes, where the ${ }^{3} \mathrm{He} /{ }^{4} \mathrm{He}$ ratio is also very high, would further test the geo-fusion hypothesis. The detection of significant naturaltritium emissions would certainly contribute to our understanding of fusion in condensed matter as well as heat formation in the earth and possibly other planets.

As J. E. Quick et al. observed in their 1991 paper: 6 "One of the most provocative and controversial assertions in the physical sciences during the last decade has been that deuteriumdeuterium fusion may occur within certain matrices (e.g., Pd and Ti) at low temperatures and pressures [Reference here to Jones et al. also to Pons and Fleischmann]. Cold fusion would have tremendous implications for geology and planetary science, because it could contribute significantly to internal heat production in planets." They also note: "If a cold-fusion-like process occurs within the Earth, than a test for its existence would be a search for anomalous tritium in volcanic emissions." "We have emphasized this test before, ${ }^{1,2,4}$ and now encourage a search for tritium in magmatic waters and gases of hot-spot volcanoes such as Kilauea, Loihi, and Icelandic volcanoes. 


\section{From Cold Fusion to Cold Nucleosynthesis}

If metal lattices enhance nuclear reactions between deuterons ${ }^{1-4}$, could not reactions between heavier elements be likewise enhanced? The Coulomb barrier is expected to grow as $\mathrm{Z}^{2}$. Yet, since fusion and nucleosynthesis both occur in stars and now that we find that fusion occurs at measurable rates in metals, we propose nucleosynthesis may also occur in metal lattices. Hence "cold nucleosynthesis" arises as a natural if bold extension of the original cold fusion hypothesis. While cold nucleosynthesis experiments are underway at BYU (involving heavier elements), we acknowledge that several other groups have made striking progress in this field as reported at this conference. Neither cold fusion nor cold nucleosynthesis is understood at present, nor are the results yet widely accepted by the scientific community. But as we continue to explore together, cooperative experiments at several laboratories giving positive results cannot be ignored much longer.

We would like to point out that anomalous results reported years ago by Ed Cecil ${ }^{7}$ and by Graham Hubler ${ }^{8}$ could possibly be due to cold nucleosynthesis. Cecil prepared deuterated samples Ti662 $(6 \% \mathrm{~V}, 6 \% \mathrm{Al}, 2 \% \mathrm{Sn})$, so that deuteron reactions on these metals should be considered. "Intense bursts of energetic charged particles from deuterium gas loaded thin foils of Ti662 subject to fairly high D.C. electric currents and non-equilibrium thermal conditions have been observed. The energies of the particles range from less than $1 \mathrm{MeV}$ to greater than 10 $\mathrm{MeV}$. By observing energy shifts through thin foils of $\mathrm{Al}$, the particles are tentatively identified as tritons, ${ }^{3} \mathrm{He}$, and possibly alpha particles. Control measurements carried out with comparably prepared Ti-H samples evinced no particle bursts." 7 Hubler reported similar anomalies: "Evidence for a high rate of production of $\mathrm{MeV}$ energy nuclear reaction products during lowenergy ion beam charging of Ti with a deuterium beam produced by an ECR plasma source is presented. According to the laws of conventional nuclear physics, nuclear reactions cannot occur at such large rates for sub-keV energy deuteron bombardment; yet we are unable to uncover a plausible artifact that could product the observed signals. As such, we believe that the implications of the data are very provocative and that this effect warrants investigation by other researchers. Further, the results... indicate that an enhancement of the charged particle count rate above background is present in the $5 \mathrm{MeV}$ energy region." ${ }^{8}$ The results were very puzzling at the time since they could not be explained in terms of d-d fusion, as I discussed the results with Ed and Graham. It is understandably very difficult to publish such results in conventional physics journals. These striking data involving deuterons in titanium were presented at the BYU conference in $1990,{ }^{7,8}$ might well be reconsidered in light of recent experiments regarding dnucleosynthesis on heavier metals. We believe both experiments represent real effects measured by competent physicists which should be appreciated and re-visited.

We were likewise impressed by the experimental results presented at this conference by Y. Iwamura, A. Takahashi, and F. Celani. ${ }^{2}$ All three groups report transmutations of the type $\mathrm{Z}+4, \mathrm{~A}+8$. Yasuhiro Iwamura and his team at the Advanced Technology Research Center of Mitsubishi Heavy Industries, Ltd., showed clear and repeatable evidence for formation of $\operatorname{Pr}$ (a rare earth) from $\mathrm{Sr}$ and $\mathrm{Mo}$ (mostly Mo-96) from Cs. A paper giving methods and earlier results is found in Jpn. J. Appl. Phys 41 (2002) 4642-4650. Others presented related "cold nucleosynthesis" effects involving hydrogen isotopes and various elements. 
Our work at BYU was stimulated early on by the above papers and by reports from Kevin Wolf (with whom we collaborated on experiments), George Miley, R. Sundaresan, and M. Singh et al. We currently employ graphite rods in our experiments along with various metals and deuterium, although it would be premature to discuss results here. We are joined in our experiments at Brigham Young University by Prof. Lawrence Rees, an expert in elemental analysis techniques such as X-ray Fluorescence (XRF) and PIXE (Proton Induced X-ray Emission). Both of these instruments are available at BYU. We also have ICP-MS and SEM as well as a new Transmission Electron Microscope (with EDS and EELS for elemental analysis). I am pleased that BYU also has a Time-of-Flight SIMS instrument so that we can perform stateof-the-art isotopic analysis in-house. For detecting gamma and X-rays, we have high-sensitivity germanium and lithium-drifted silicon detectors. Of course, sensitive neutron detectors are also available at BYU.

To evaluate charged particles emitted during nucleosynthesis, we plan to use ionimplanted silicon detectors. In particular, we anticipate the probable formation of energetic alpha particles during such nucleosynthesis reactions (if they occur). A great deal of information can be gleaned from the observation of any nuclear particles produced during cold nucleosynthesis reactions. Knowing the identities and energies of any emitted particles would provide confirmation and enormous insight into what is taking place. So our goal is to measure elements/isotopes before and after as others have done - and to measure the energies of emitted nuclear particles while these strange reactions are taking place.

We invite other researchers in this field to send samples to BYU for analysis if you wish, just as we have provided use of our neutron detection facility in the past and wish to continue to encourage this research. There are many potential applications for cold fusion and cold nucleosynthesis if the processes can be verified, understood, and enhanced, so we continue our research in these closely-related areas.

\section{REFERENCES}

[1] C. DeW. Van Siclen and S. E. Jones, "Piezonuclear fusion in isotopic hydrogen molecules," J. Phys. G: Nucl. Phys. 12: 213-221 (March 1986).

[2] S. E. Jones, E. P. Palmer, J. B. Czirr, D. L. Decker, G. L. Jensen, J. M. Thorne, S. F. Taylor, J. Rafelski, Observation of Cold Nuclear Fusion in Condensed Matter, Nature 338: 737-740 (April 1989).

[3] See S. E. Jones et al., "Nuclear Emissions from Metal Deuterides," in this Proceedings.

[4] S.E. Jones, et al., "Anomalous Nuclear Reactions in Condensed Matter: Recent Results and Open Questions,” J. Fusion Energy, 9:199 - 208 (1990). 
[5] F. Goff and G. M. McMurtry, "Tritium and stable isotopes of magmatic waters," J. Volcanology and Geothermal Research, 97: 347-396 (2000); F. Goff, G. M. McMurtry, D. Conce, J. A. Simac, A. R. Roldan-Manzo, D. R. Hilton, "Contrasting Hydrothermal activity at Sierra Negra and Alcedo volcanoes, Galapagos Archipelago, Ecuador," Bull. Volcanol. 62: 34$52(2000)$.

[6] J. E. Quick, T.K. Hinkley, G.M. Reimer and C.E. Hedge, "Tritium concentrations in the active Pu'u O'o crater, Kilauea volcano, Hawaii: Implications for cold fusion in the Earth's interior," Physics of the Earth and Planetary Interiors, 69: 132-137 (1991).

[7] F. E. Cecil, H.Liu, D. Beddingfield, and C. S. Galovich, Observation of Charged-particle Bursts from Deuterium-Loaded Thin-Titanium Foil, American Institute of Physics: Conference Proceedings 228, NY (1991, S.E Jones, F. Scaramuzzi, \& D. H. Worledge, eds.). See Chapter 2, Charged-Particle Emissions, Anomalous Nuclear Effects in Deuterium/Solid Systems, p. 375

[8] G. P. Chambers, G. K. Hubler, and K. S. Grabowski, Search for Energetic ChargedParticle-Reaction Products During Deuterium Charging of Metal Lattices, American Institute of Physics: Conference Proceedings 228, NY (1991, S.E Jones, F. Scaramuzzi, \& D. H. Worledge, eds.). See Chapter 2, Charged-Particle Emissions, Anomalous Nuclear Effects in Deuterium/Solid Systems, p. 383 